\title{
Nanoscale Mass Spectrometry Multimodal Imaging via Tip-Enhanced Photothermal Desorption
}

\author{
Matthias Lorenz, ${ }^{\dagger,+*}$ Ryan Wagner, ${ }^{\S, \#}$ Stephen Jesse, ${ }^{\dagger}$ Jennifer M. Marsh," Marc Mamak," Roger \\ Proksch, $§$ Olga S. Ovchinnikova ${ }^{\dagger, *}$ \\ ${ }^{\dagger}$ Center for Nanophase Materials Sciences, Oak Ridge National Laboratory, Oak Ridge, \\ Tennessee 37831, United States \\ University of Tennessee, Knoxville, Tennessee 37996, United States \\ $\S$ Asylum Research an Oxford Instruments Company, Santa Barbara, California 93117, United \\ States \\ "Procter \& Gamble Company, Cincinnati, Ohio 45202, United States
}

* Author to whom correspondence should be addressed.

Matthias Lorenz

Center for Nanophase Materials Sciences

Oak Ridge National Laboratory

1 Bethel Valley Rd

Oak Ridge TN, 37831-6493

lorenzm@utk.edu

Olga S. Ovchinnikova

Center for Nanophase Materials Sciences

Oak Ridge National Laboratory

1 Bethel Valley Rd

Oak Ridge TN, 37831-6493

ovchinnikovo@ornl.gov

\# Present address: School of Mechanical Engineering, Purdue University, West Lafayette, Indiana 47907, United States

Notice: This manuscript has been authored by UT-Battelle, LLC, under Contract No. DEAC0500OR22725 with the U.S. Department of Energy. The publisher, by accepting the article for publication, acknowledges that the United States Government retains a non-exclusive, paidup, irrevocable, world-wide license to publish or reproduce the published form of this manuscript, or allow others to do so, for the United States Government purposes. The Department of Energy will provide public access to these results of federally sponsored research 
in accordance with the DOE Public Access Plan (http://energy.gov/downloads/doe-publicaccess-plan). 


\section{Additional Experimental Details}

\section{Set-up}

The modification of the ES cell body made it necessary to remove an $\sim 10 \mathrm{~mm}$ wide section of the supporting ring structure on the ES scanner to accommodate the ES cell with the tube port attached.

The positive edge of a trigger sync signal coming from the mass spectrometer indicating the beginning of the Injection Period of the FT (Orbitrap) mass analyzer was passed through a lowpass RC filter circuit with a time constant of $\tau=10 \mu \mathrm{s}(10 \mathrm{k} \Omega, 1 \mathrm{nF})$ and fed into the Trigger In channel of a National Instruments (Austin, TX) PXIe-6124 DAQ board, equipped with a BNC2110 breakout box and accommodated in a PXI chassis. The DAQ board was programmed using custom-built software developed in National Instruments LabVIEW 2016 on a personal computer running Microsoft (Redmond, WA) Windows 7. A square pulse of variable timing, duration and amplitude was provided to a BNC analog input (BNCIn0) on the Cypher AFM. The analog signal of $0-10 \mathrm{~V}$ was relayed to the blueDrive controller using settings in the Crosspoint panel of the

control software for the microscope (connecting BNCIn0 and bDDrive virtual ports). A second TTL trigger pulse with synchronized rising edge but longer duration than the pulse controlling the laser diode was fed into the MS on an A/D input channel.

\section{General workflow and details}

Switching between contact mode AFM and TD further required the change of the blueDrive driver source (external BNCIn0 to DDSA) through the Crosspoint panel of the control software of the microscope.

The MS was set to continuously record mass spectra (chronograms) for the time of the TD imaging experiments, resulting in the acquisition of spectra with a period that was mainly dictated 
by the scan time of the mass analyzer. A second TTL trigger pulse with synchronized rising edge but longer duration than the pulse controlling the laser diode was fed into the MS on a A/D input channel. This channel would provide an estimate of the timing of the laser trigger events.

Mass spectra for the entire course of an experiment were exported as ASCII files, converted to constant width $(5 \mathrm{mDa}) \mathrm{m} / \mathrm{z}$ bins and filled with zero values, and then saved in an HDF file container. This was performed using software developed in house in Python 3, using the NumPy library and the h5py package to provide an interface to the HDF5 (hierarchical data format) file format.

\section{Discussion of Figure 1}

The gas flow configuration of the inline APCI source consist of two orthogonal gas flows entering the metal cross - the desorption gas flow traveling through the flow cell, and the additional, needle-valve adjusted gas feed that is used to dilute and adjust the aspiration of gas from the desorption cell. The balancing of the two gas inflows into the metal cross fitting with the outflow critically affects the transport into the AP mass spectrometer, hence the pressure in the first pressure reduced stage, e.g., in the vacuum recipient behind the ion transfer tube. It can be assumed that an excess supply of nitrogen, which is beneficial for the operation of the inline APCI source (cf. Fig. 1d), reduces the amount of air aspirated into (and through) the desorption cell. The signal increases even though less analyte material is sampled. It should be noted that the AP inlet and pressure reduction cascade is designed and optimized to work freely aspirating from AP which is indicated by the dashed line in Figure 1d.

With a gas flow of about $800 \mathrm{~mL} / \mathrm{min}(1.6 \mathrm{~L} / \mathrm{min}$ total inflow into ion transfer tube, diluted $1 / 2$ by introduction of dry nitrogen at the inline APCI stage) with the given tubing dimensions of 
sampling tube, PFA tubing, and the ion transfer tube, a total transfer time of 9 ms can be estimated for the transport from the desorption site to the end of the ion transfer tube in the first vacuum recipient of the AP mass spectrometer (cf. paragraph Transfer time for desorbed material under Calculations). 


\section{Calculations}

Transfer times for desorbed material

Dimensions of sampling tube parts:

Sampling tube: $700 \mu \mathrm{m}$ i.d., $50 \mathrm{~mm}$ length

PFA tubing: $950 \mu \mathrm{m}$ i.d., $90 \mathrm{~mm}$ length

Ion transfer tube: $580 \mu \mathrm{m}$ i.d., $59 \mathrm{~mm}$ length

Total volume of tubing:

$$
V=\frac{\pi}{4}\left(d_{1}^{2} l_{1}+d_{2}^{2} l_{2}+d_{1}^{2} l_{3}\right)=\frac{\pi}{4}(24.50+81.23+19.85) \mathrm{mm}^{3}=121.3 \mathrm{~mm}^{3}
$$

Estimation of purge time for total transfer line (for $800 \mathrm{~mL} / \mathrm{min}$ aspiration flow rate):

$$
t=\frac{V}{\phi}=\frac{121 \mathrm{~mm}^{3}}{800 \mathrm{mLmin}^{-1}}=\frac{121 \mathrm{~mm}^{3}}{13333 \mathrm{~mm}^{3} \mathrm{~s}^{-1}}=9.1 \mathrm{~ms}
$$

Volume of smallest amount of detected thermally desorbed material

Dimensions of a conical crater volume (this work):

Base diameter: $887 \mathrm{~nm}$

Height: $156 \mathrm{~nm}$

$$
V_{\text {cone }}=\frac{\pi}{12} d^{2} h=\frac{\pi}{12}\left(1.23 \times 10^{8} \mathrm{~nm}^{3}\right)=3.2 \times 10^{7} \mathrm{~nm}^{3}
$$

Dimensions of a conical crater volume (previous work):

Base diameter: $1000 \mathrm{~nm}$

Height: $600 \mathrm{~nm}$

$$
V_{\text {cone }}=\frac{\pi}{12} d^{2} h=\frac{\pi}{12}\left(6.00 \times 10^{8} \mathrm{~nm}^{3}\right)=1.6 \times 10^{8} \mathrm{~nm}^{3}
$$




\section{Figures}

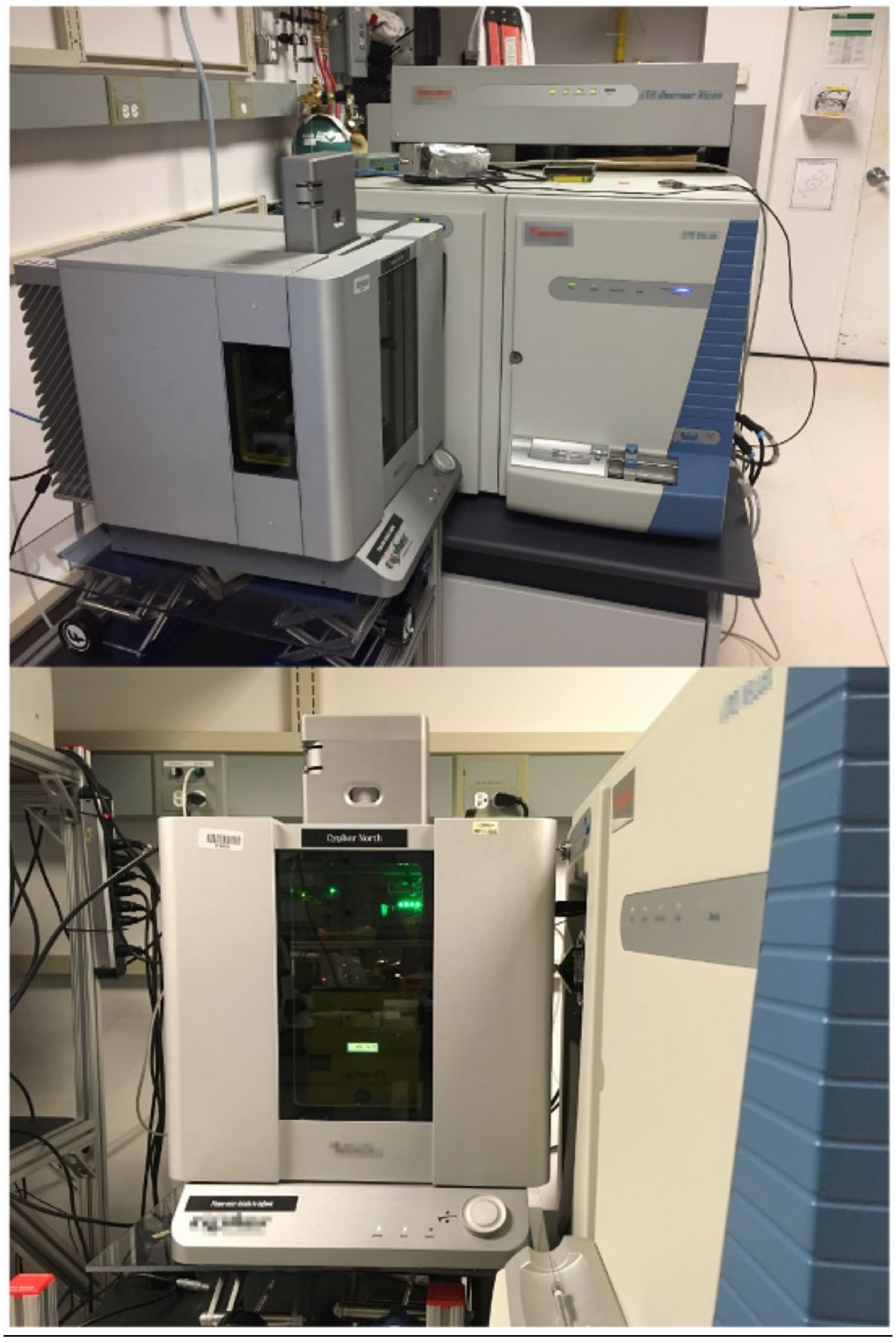

Figure S1. Photograph of the set-up. A stable board made of acrylic glass, elevated and height adjusted using lab jacks on a cart, was used to support the AFM. No vibration isolation stage was 
used. Care was taken to prevent any avoidable physical contact with the LTQ Orbitrap Velos Pro and to restrict the contact to the transfer tubing.

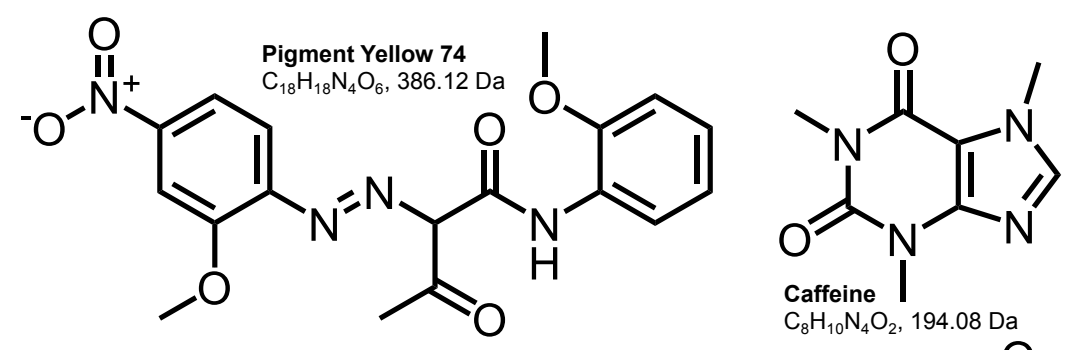

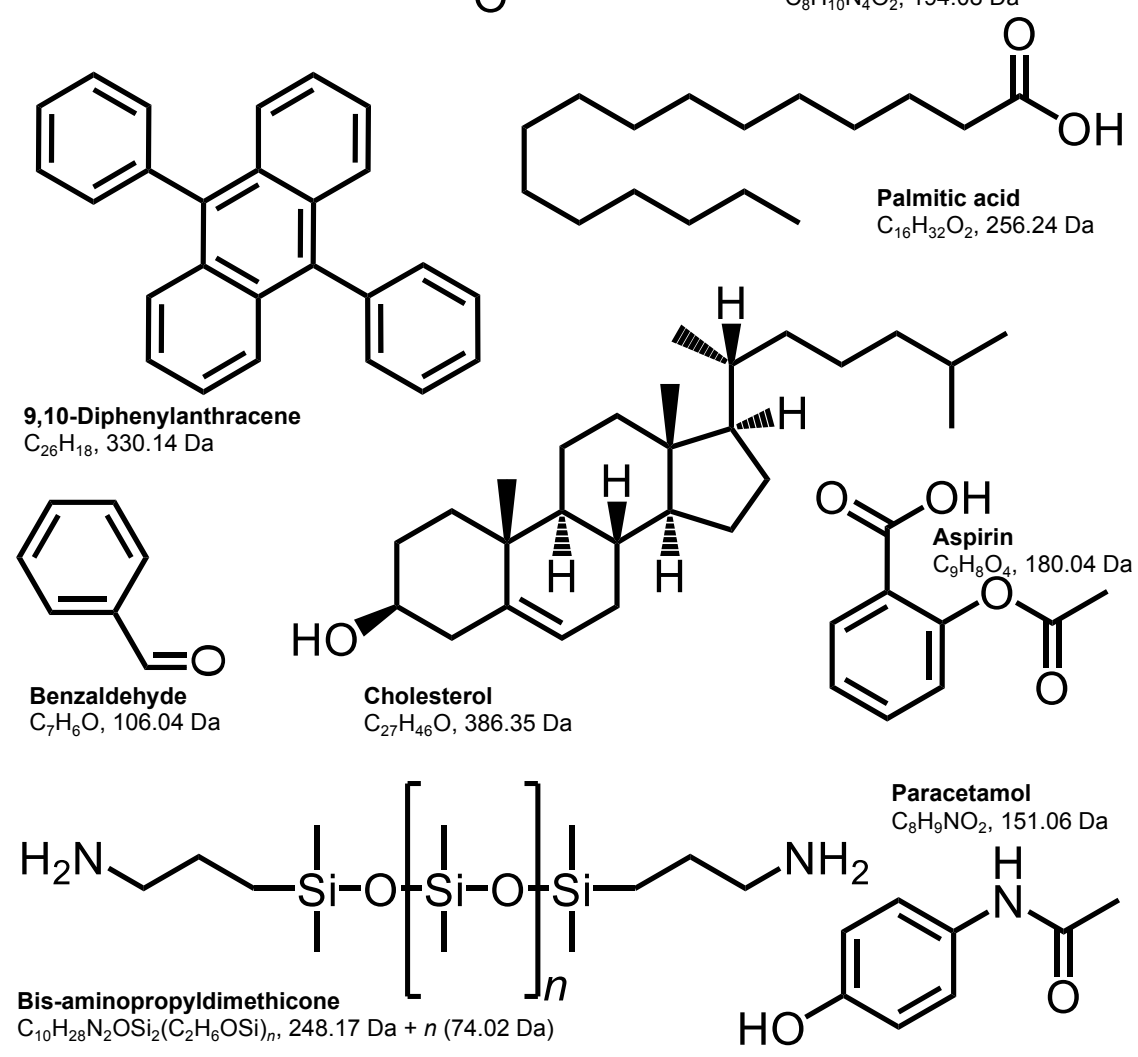

Figure S2. Chemical structures. Shown are structures of the analyte molecules desorbed in contact with the photothermally heated AFM tip and ionized by inline APCI in positive ion mode. 

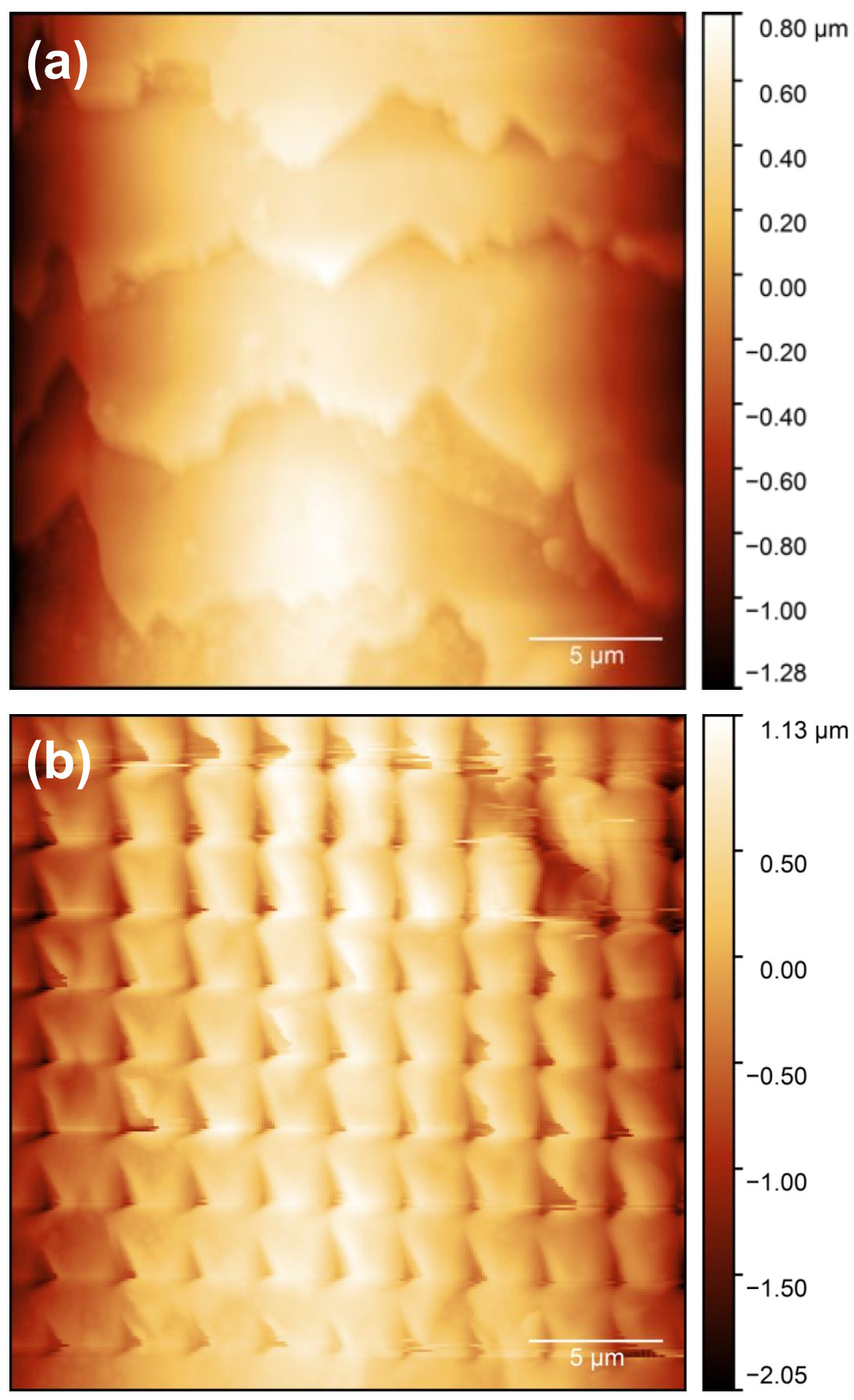

Figure S3. Hair sample prior vs. after AFM-MS analysis. Shown are AFM images of the human hair sample studied in Fig. 5, (a) before and, (b) after mapping with TD for AFM-MS analysis. 

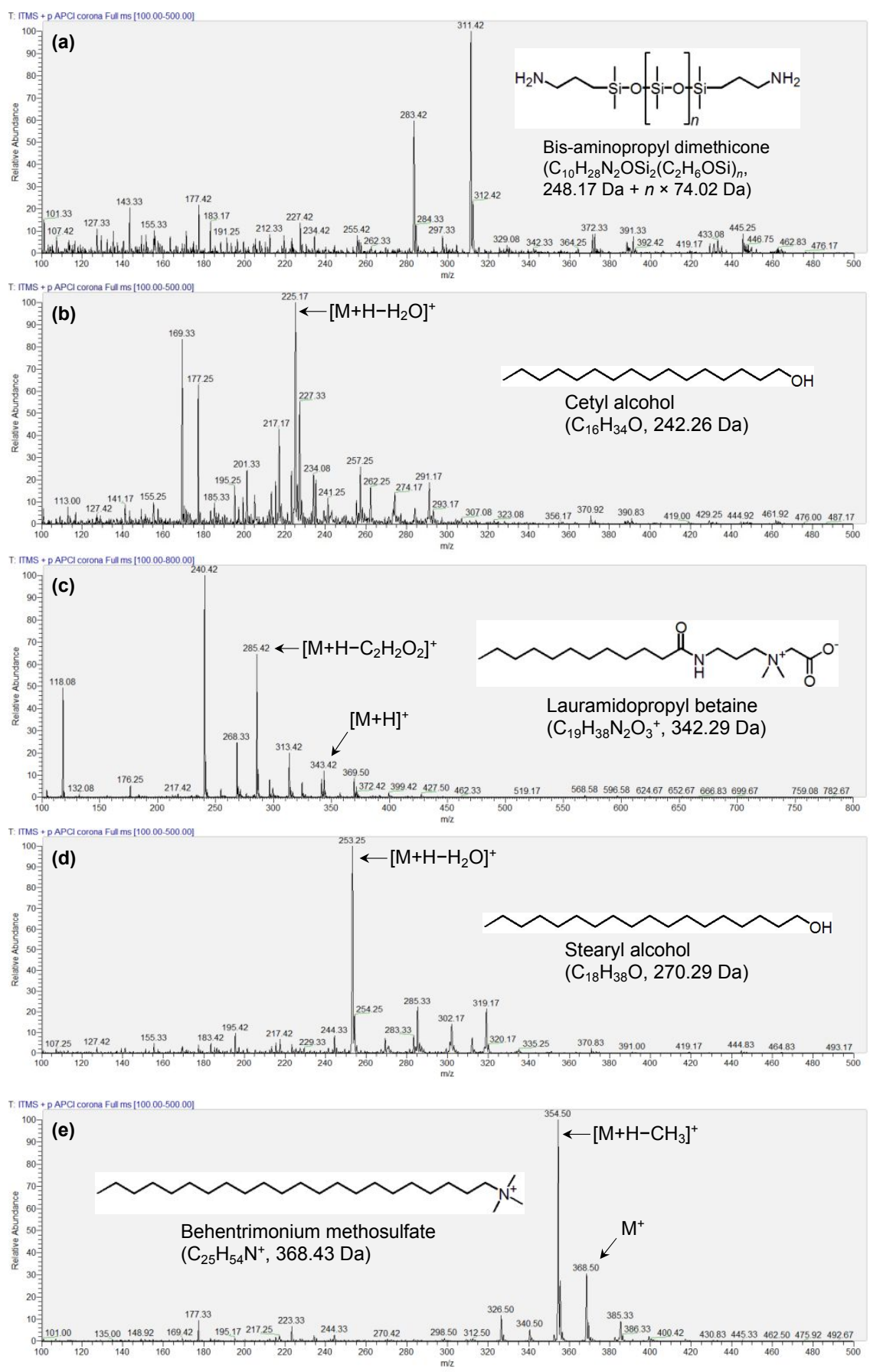

Figure S4. Spectra of standards. Reference mass spectra have been recorded for some active ingredients contained in the hair care product applied to the sample used in Fig. 5. Data were recorded on an AFM-MS platform using a resistively heated AFM probe and a Thermo Fisher Scientific LTQ XL mass spectrometer with linear iontrap detector (unit mass resolution). 


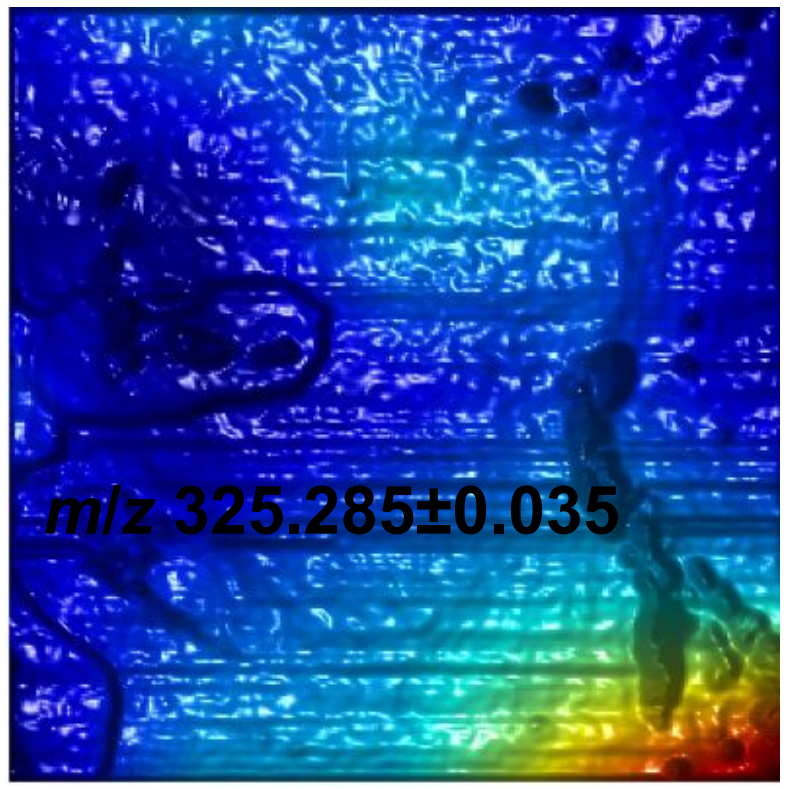

Figure S5. AFM-MS multimodal analysis of sticky note product. Figure shows additional normalized ion image for given $\mathrm{m} / \mathrm{z}$ interval color-encoded as overlay on top of AFM phase (cf. Fig. 6). 